\title{
Decision Tree Method Using for Fetal State Classification From CARDiotography Data
}

\author{
Md. Zannatul ARIF ${ }^{1, *}$, Rahate AHMED ${ }^{2}$, Umma Habiba SADIA ${ }^{3}$, \\ Mst Shanta Islam TULTUL ${ }^{4}$, Rocky CHAKMA ${ }^{5}$
}

\author{
${ }^{1}$ Department Of Electrical Power Systems And its Automations, North China Electric Power \\ University, Changping Beijing China \\ ${ }^{2}$ Department of Nuclear and Energy Engineering, Jeju National University, Korea \\ ${ }^{3}$ Department Of Enterprise Management, North China Electric Power University, China \\ ${ }^{4}$ Dept. Of Microbiology \& Immunology, Jeju National University, Korea \\ ${ }^{5}$ Lecturer, Dept. of Electrical and Electronic Engineering, University Of Science \& Technology \\ Chittagong, Bangladesh
}

*Corresponding Author: Md. Zannatul ARIF (Email: mdzannatularif@yahoo.com)

(Received: 15-Dec-2019; accepted: 13-March-2020; published: 31-March-2020)

DOI:http://dx.doi.org/10.25073/jaec.202041.273

\begin{abstract}
The motive of the investigation is analyzing the categorization of fetal state code from the Cardiographic data set based on decision tree method. Cardiotocography is one of the important tools for monitoring heart rate, and this technique is widely used worldwide. Cardiotocography is applied for diagnosing pregnancy and checking fetal heart rate state condition until before delivery. This classification is necessary to predict fetal heart rate situation which is belonging. In this paper, we are using three input attributes of training data set quoted by $L B, A C$, and $F M$ to categorize as normal, suspect or pathological where NSPF variable is used as response variable. After drawing necessary analyzing into three variables we get the 19 nodes of classification tree and also we have measured every single node according to statistic, criterion, weights and values. The Cardiotocography Dataset applied in this study are received from UCI Machine Learning Repository. The dataset contains 2126 observation instances with 22 attributes. In this experiment, the highest accuracy is $98.7 \%$. Overall, the experimental results proved the viability of Classi-
\end{abstract}

fication and Regression Trees and its potential for further predictions.

\section{Keywords}

Decision tree, carditotcography, fetal state. FHR.

\section{Introduction}

Cardiotocography (CTG) is a universal popular process for fetal observation and is the most commonly used for evaluation of fetal state. The alternative name of CTG is Electronic Fetal Monitoring (EFM). The transducers measure fetal heart rate (FHR) is placed on the skin of the abdomen of a pregnant. Simultaneous recording of FHR are found from cardiotocogram (CTG). It catches observation the constrictions of the pregnant woman and various types of fetal monitoring too. Prenatal CTG can be an effective method of fetal evaluation during pregnancy and it tests the fetal heart rate as an indicator of the baby's primary biological activities [1]. Usually CTG is used during the last 3 months that 
means after the 28th week of pregnancy. The main background of CTG is an observation for the diagnosis of babies' fetal situation and getting information of that infants who are under the risk of having enough amount of oxygen reaching deficiency in the tissues. Fetal heart rate is the most exploited information and can't be measured directly during pregnancy because of fetus innate inaccessibility. Necessarily, clinicians depend on indirect symptoms and information to learn about fetal condition [2].

Every parent wants a healthy baby with normal delivery. It totally depends on regular care during the delivery period. In pregnancy time every mother needs to maintain sound care, intense rules and regulations for having a healthy pregnancy with a healthy delivery. During this whole period another good practice for family members is to give the full mental support to would-be mothers for preparing mentally strong and ascertaining the appropriate delivery method based on the results of CTG. If CTG examination shows the baby's condition looking good then it will be easier to take any further decisions and minimize the health risks at childbirth. Sometimes child born without a heartbeat caused by hypoxia but now the incidence of birth asphyxia is minimized because the technique of CTG. However, Caesarean section births are increased as a side effect of this method [3].

The Benefits of Cardiotography depend on the result of fetal heart rate types. Because this result data shows the clinical practice whether is it low standard or high standard? Even there is manual for taking cardiotocography data set. Both the inter-observer and intra-observer check the variation of CTG interpretation but manual interpretation sometimes increases error. To eliminate human mistake, data mining techniques are essential to examine and align the CTG data [4].

Reasonable errors need to be reduced to improve CTG analysis. A more objective approaches are highly important for CTG interpretation; hence, significant efforts have been spent in recent years and consequently, various analysis methods have been proposed [5]. As example we will notice that some automated software helps clinicians by providing signal- processing facilities to determine and measure some employed parameters like vibrancy, mutability, stimulation of fetal heart rate, frequency of UC etc. Although, the role of these computerized classification processes have only partially reduced the inter-and intra-observer variations [6].

For the fetus, the accepted parameters of Baseline fetal heart rate are started in between 110 and 160 where variance is greater than 5 [7]. Clinicians find information like continual heart rate indication and fetal heart rate variations in response of CTG. It is one kind of indirect screening test on fetal acid-base balance and is regarded as an indicator of fetal welfare [8]. Since its launch in the 1970 s, CTG is widely used in daily practice. Clinicians expected that fetal heart rate monitoring would work as a screening test for acute asphyxia which is enough to cause of neurologic damage or death of newborn $[9,10]$.

While it's practically, the effectiveness and success of CTG monitoring has some inconsistencies, specifically in low-risk pregnancies. If fetal pain is examined inaccurately, it may be mentioned to as needless treatments. On the other hand, an inappropriate analysis of fetal well-being may be refused necessary treatments [11].

The classification is used to measure performance evaluation, actually it's not enough to make a conclusion for such an important situation as the field of medical diagnosis. Because of that, it is proposed another kind of implement measurement tools as like the decision tree. In this paper, our vision is to analyze CTG data set based on fetal classification code by using decision tree method.

Decision tree (DT) is a process to constitute an alignment model. Like the name it suggests, a tree-shaped form is built using inductive argument. Decision tree has turned to be a very fruitful appliance for performing medical prophecy models [12].

Intense outcome is caused by the embryo because of fetal distress. The purpose of this research is to establish a classification model that has maximum possible precision to subsequently 
assist gynecologists in the diagnosis of embryology [13].

The paper has been divided into some parts as follows. At first talks about training data set, $2^{\text {nd }}$ part is about proposed methodology and $3^{\text {rd }}$ part contains the sampling dataset. $4^{\text {th }}$ part explains different models and results. In part 5 , we design about results discussion and work preview.

\section{Training data set}

In this study, mentioned cardiotocography data set has taken from "The Data Mining Repository of University of California Irvine (UCI)" [14]. This training data set has consisted of 2126 samples fetal cardiotocograms which belongs to different classes with 21 attributes and two class labels.

In this training, we used fetal state class code as object point attribute in exchange to fetal heart rate sampling group and each group has illustrated into three different types as normal, suspect and pathological.

The Attribute information is given Tab. 1.

\section{Proposed methodology}

Classification and regression Trees is called CART or Tree. CART is one of the renowned supervised learning algorithms. It is a choice support tool that is employed as a tree-like pattern of selections and their possible consequences, resource values, and utility. This is a way of displaying algorithms that are only represented as a binary tree.

Decisions trees are usually utilized in research, to assist determine the technique possible to achieve a goal, however also are a well-liked tool in machine learning algorithm.

Classification and regression trees (CART) regarded as a decision tree technic employed for classification motive using the diachronic data. As a machine learning method, it is introduced in 80 s by and is considered to ascertain number of classes [15]. To form decision trees, CART requires learning sample. Decision trees are a set of questions, which is divided the learning example into short and unconsidered parts. In order to acquire the best divide, the query that divides the data into 2 kindred portions, CART system explores of all probable variables and values [16].

CART is a binary decision tree approach and shows the direction for another algorithms like Random Forest, Bagged Trees and Boosted trees, where every root node explains a single input variable and a divide point on that variable. The leaf nodes comprise the response variable which is used to establish predictions [17]. CART uses Gini index to pick out the attribute which has maximum info. A data "A" with $n$ categories has Gini Index that's outlined as

$$
\operatorname{Gini}(a)=1-\sum_{1}^{n} p j^{2}
$$

Knowing the learning system is necessary due to understand which groups exits in a given training data set. Moreover, there is a vector of attribute values for every system and a mapping function to assemble groups from the attribute values [18]. The features applied to the defined examples can be combined into continuous features with ungraded nominal values [19]. Here C4.5 can be taken as an instance of that system which instructs decision-tree classifiers method [20].

For getting new measurement here we utilize c4.5 that is also known as Gain ratio. It can be described by below formula,

$$
\operatorname{Entropy}(p)=1-\sum p j \log (p i)
$$

$\operatorname{Gain} \operatorname{Ratio}(p, T)=\operatorname{Gain}(p, T) / \operatorname{Split} \operatorname{Infor}(p, t)$

When a decision tree has been formed, in some branches exceptions in the training data will appear because of outliers or noise. To exceed data over fitting matter, tree pruning approach can be used by employing statistical measures to eject the maximum uncertain branches. Pre-pruning and post-pruning are the common tree pruning 
Tab. 1: Training Data Set

\begin{tabular}{|c|c|}
\hline Features Symbol & Attribute description \\
\hline LB & FHR baseline (beats every minute) \\
\hline $\mathrm{AC}$ & accelerations every second \\
\hline FM & fetal movements every second \\
\hline$\overline{\mathrm{UC}}$ & uterine constrictions every second \\
\hline$\overline{\mathrm{DL}}$ & light decelerations every second \\
\hline$\overline{\mathrm{DS}}$ & severe decelerations every second \\
\hline$\overline{\mathrm{DP}}$ & prolongued decelerations every second \\
\hline ASTV & $\%$ of time with abnormal short term variability \\
\hline MSTV & mean value of short term variability \\
\hline ALTV & $\%$ of time with abnormal long term variability \\
\hline MLTV & mean value of long term variability \\
\hline Width & width of FHR histogram \\
\hline Min & minimum of FHR histogram \\
\hline $\operatorname{Max}$ & Maximum of FHR histogram \\
\hline Nmax & histogram peaks \\
\hline Nzeros & histogram zeros \\
\hline Mode & histogram mode \\
\hline Mean & histogram mean \\
\hline Median & histogram median \\
\hline Variance & histogram variance \\
\hline Tendency & histogram tendency \\
\hline CLASS & FHR pattern class code \\
\hline NSP & fetal state class code \\
\hline
\end{tabular}

**Data Source: University of California Irvine (UCI).

techniques [21, 22]. In a decision tree, data classification has two-phase operation where training phase is the first one, and another one is classification phase. At first phase, a training data is assisted for composition of the tree and trees rules are ascertained in accordance with this training data [23].

In this thesis for sampling training data set we follow a conceptual process. The experimental Sampling Data Set Process model is given by Fig. 1.

\section{Sampling data set}

We have used Cardiotocogram data set which is divided into 21 attributes, and another one is response variable. For this data set we have used input data accordingly LB, AC and FM at- tribute. At first, we need to sampling and processing our data set. In this experiment, we have used $\mathrm{R}$ programming tools for getting our desire result. The raw data structure is below -

This data frame is 2126 observations of 22 variables. But this 22 variables accordingly LB, ASTV, ALTV, Width, Min, Max. Nmax, Nzeros, Mode, Mean, Median, Variance, Tendency and NSP are integer. Rest of the Ac, FM, UC, DS, DP, MSTV are numerical.

Mentioned data structure types are integer and numerical variable. In this data structure 13 are integer variable and rest of the 8 are numerical out of 21 features. Last variable NSP is response variable which one is numerical and coded as 1, 2 and 3. Depending whether classification list is Normal, suspect and pathological. In $R$ programming reads it is factor or categorical variables. For this reason we need to change 


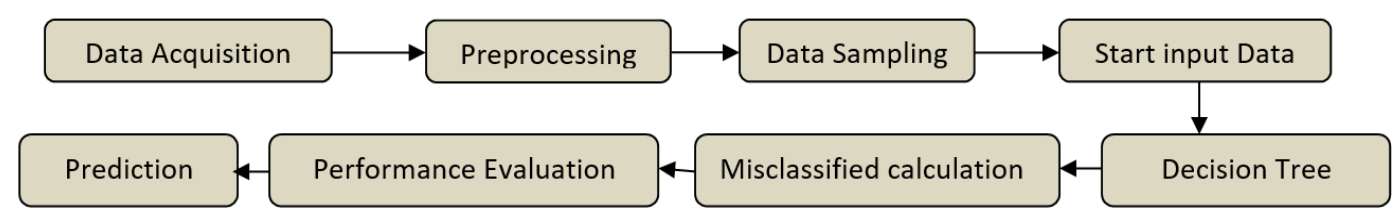

Fig. 1: A conceptual model of the proposed approach.

this response variable. After input the data set it will be like below -

NSPF : Factor w/3 levels "1","2","3": 2111 13 Add another new attribute in total 23. New variable name is NSPF which one detect in $R$ programming as a factor variables. Finished the reform of data set we did allocation data into training and validation data set.

After divide the data set will be Cardiotocographic, Sampling data, learned and verified data as seen in Tab. 2

Tab. 2: Sampling data set measurement

\begin{tabular}{|c|c|c|c|}
\hline $\begin{array}{c}\text { Number } \\
\text { of } \\
\text { contents }\end{array}$ & $\begin{array}{c}\text { Data } \\
\text { Set name }\end{array}$ & $\begin{array}{c}\text { Total } \\
\text { obser } \\
\text {-vation }\end{array}$ & $\begin{array}{c}\text { Total } \\
\text { variables }\end{array}$ \\
\hline 01 & $\begin{array}{c}\text { Cardioto } \\
\text {-cographic }\end{array}$ & 2126 & 22 \\
\hline 02 & $\begin{array}{c}\text { Sampling } \\
\text { Data }\end{array}$ & 2126 & 23 \\
\hline 03 & Trained & 1718 & 23 \\
\hline 04 & Validated & 408 & 23 \\
\hline
\end{tabular}

\section{Results}

For finding decision tree classification we have used three input variables which are $\mathrm{LB}, \mathrm{AC}$ and FM while NSPF represents as response variable. Input data is trained data set, formed with total 1718 observations. After experiment into three variables we get 19 nodes of classification tree. Every single node is showing measurement according to statistic, criterion, weights and values.

\section{Response: NSPF}

Inputs: $\mathrm{LB}, \mathrm{AC}, \mathrm{FM}$

Number of observations: 1718
1) $\mathrm{AC} \leq 0.000834028 ;$ criterion $=1$, statistic $=$ 263.403

2) LB $\leq 136$; criterion $=1$, statistic $=131.511$

3) $\mathrm{FM} \leq 0.111898 ;$ criterion $=1$, statistic $=$ 35.729

$4)^{*}$ weights $=405$

3) $\mathrm{FM}>0.111898$

$5)^{*}$ weights $=11$

2) $\mathrm{LB}>136$

$6)^{*}$ weights $=314$

1) $\mathrm{AC}>0.000834028$

7) $\mathrm{AC} \leq 0.002209945 ;$ criterion $=1$, statistic $=$ 52.155

8) $\mathrm{LB} \leq 136 ;$ criterion $=0.999$, statistic $=$ 17.292

9) $\mathrm{FM} \leq 0.0121396 ;$ criterion $=1$, statistic $=$ 42.826

$10)^{*}$ weights $=103$

9) $\mathrm{FM}>0.0121396$

$11)^{*}$ weights $=7$

8) $\mathrm{LB}>136$

$12)^{*}$ weights $=78$

7) $\mathrm{AC}>0.002209945$

13) LB $\leq 110$; criterion $=1$, statistic $=18.889$

$14)^{*}$ weights $=18$

13) $\mathrm{LB}>110$

15) $\mathrm{LB} \leq 147 ;$ criterion $=0.965$, statistic $=$ 8.877

16) $\mathrm{FM} \leq 0.2354892$; criterion $=0.986$, statistic $=10.725$
$17)^{*}$ weights $=742$
16) $\mathrm{FM}>0.2354892$
$18)^{*}$ weights $=12$
15) $\mathrm{LB}>147$
$19)^{*}$ weights $=28$

This classification of tree represented by Fig. 2 .

The tree (in Fig. 2) generally grows upwards and downwards. If we look at the graph, roots are at the top and leaps are bottom. Basically most important roots will be stand at top position of prediction models. In this classification most important variable is AC, that's why its position is above and each of the three variables helps to classify 1,2 and 3 which 


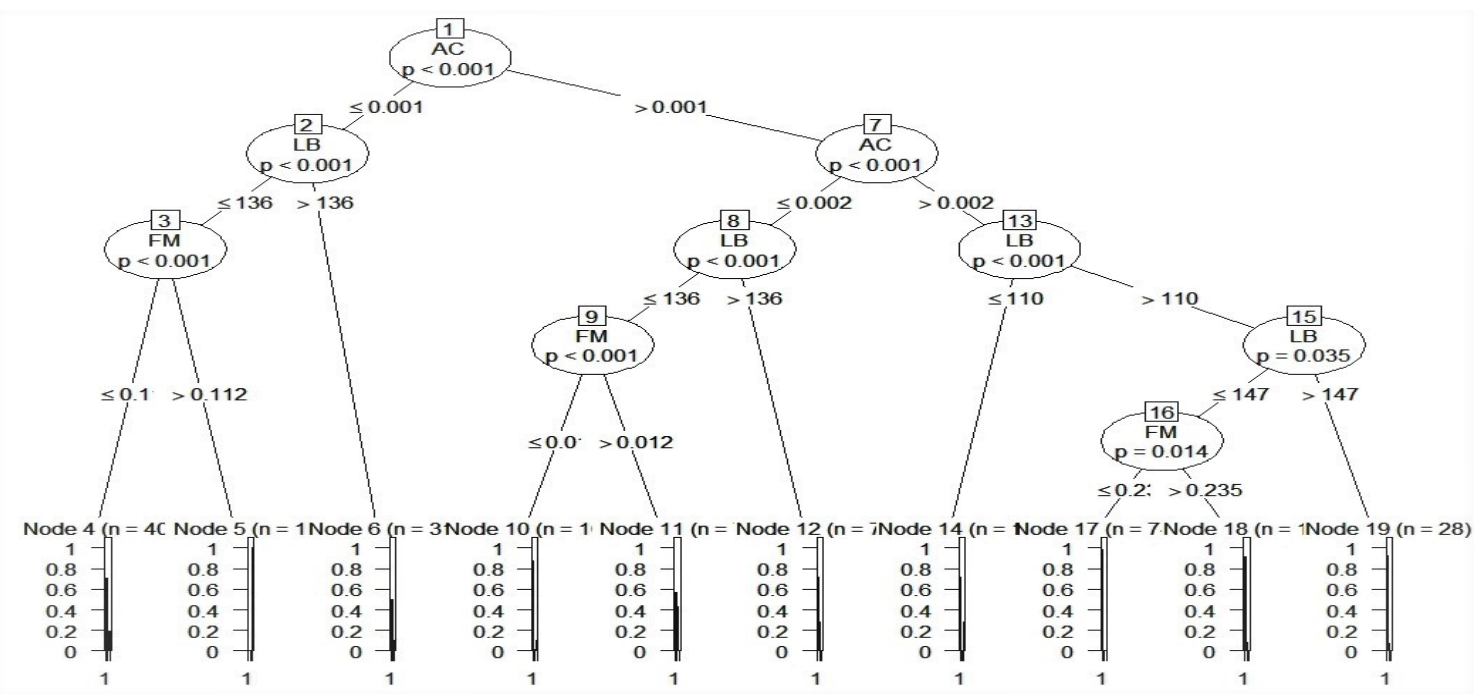

Fig. 2: Classification of Decision Tree.

are represented normal, suspect and pathological. If $\mathrm{AC}$ is $<0.001$, it will go to left side and if more than $>0.001$, it will go on right side. Similarly if LB is $<136$, will go to left side and more than $>136$ will go on right side. Final variables FM is $<0.1$ which will go terminal node and this is the decision tree. Our total validate data set has 408 observations. For predict into the validation data set based on the tree we will use this 408 observations data. We had trained this data for getting our experimental results that how much probability to get normal, suspect and pathological. The results of this data prediction are given below -

[[398]]

[1] 0.692307690 .088942310 .21875000

[[399]]

[1] 0.9859335040 .0076726340 .006393862

[[400]]

[1] 0.9859335040 .0076726340 .006393862

[[401]]

[1] 0.692307690 .088942310 .21875000

[[402]]

[1] 0.692307690 .088942310 .21875000

[[403]]

[1] 0.692307690 .088942310 .21875000

[[404]]

[1] 0.692307690 .088942310 .21875000

[[405]]

[1] 0.692307690 .088942310 .21875000
[[406]]

[1] 0.692307690 .088942310 .21875000

[[407]]

[1] 0.40127390 .49681530 .1019108

[[408]]

[1] 0.40127390 .49681530 .1019108

Here, we just represented only last 10 data results out of 408 observations. If we look at predict data, we can see there are three types of categories, 1st one is showing probability of patients will be normal, similarly 2nd one is suspected and last one is pathological. We can also use this validate data set for prediction without probability. We have used rpart packages for validate data based on decision tree. Graphically it will be Fig. 3 .

Figure 3 shows that predict sample accordingly three variables. If we look the $\mathrm{AC}$ then we will see 934 patients are normal, 33 is suspect and 21 is pathological. Similar to others two features. Here we used extra 1 for seeing the result that how its show the graphically and same to way we used the extra value 2, 3 and 4 so it will show changes. Again if we used the extra two then it will be show differences. The different graph is given Fig. 4.

\section{Discussions}

In this train data set we get classification error is 0.19798. And out of 408 validate data observation we get the classification error is 0.2107 . 


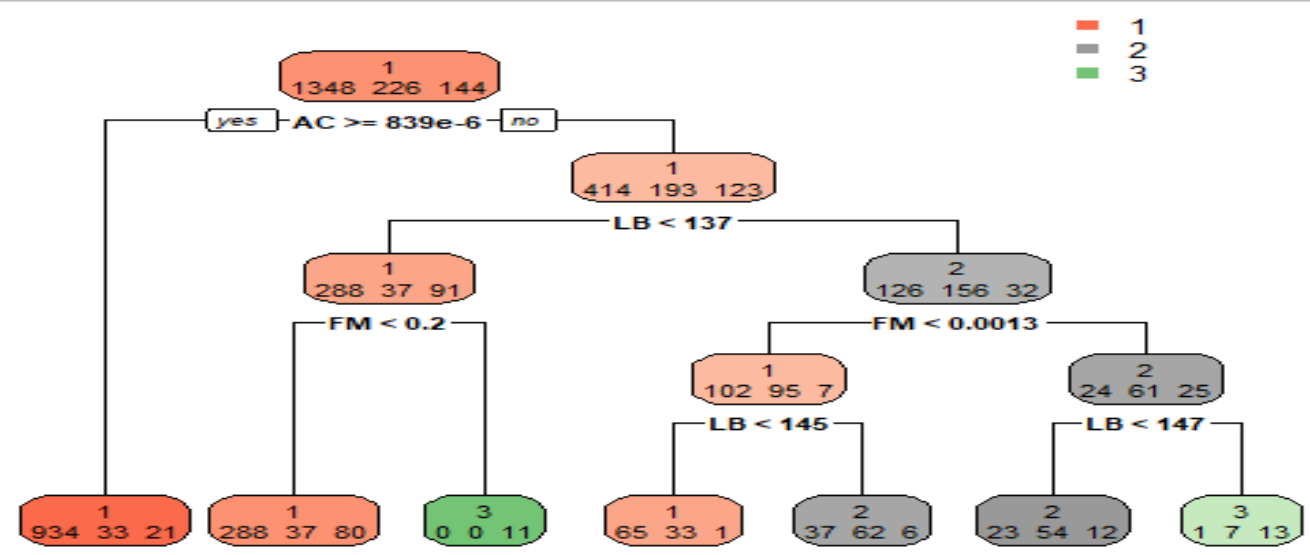

Fig. 3: Decision Tree Classifications based on Validate data.

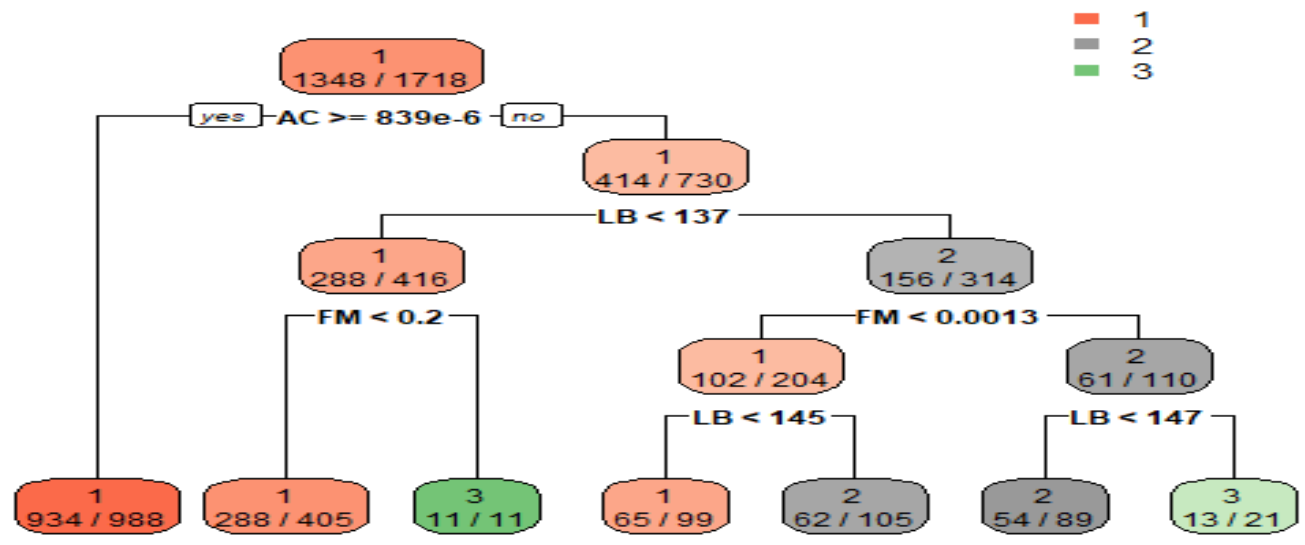

Fig. 4: Decision tree Classification based one validate data $\left(2^{\text {nd }}\right)$

If we difference the both data set then we can see this classification error is small. Out of 2126 samples data records, 1655 samples are included as normal group and 295 samples belongs to suspicious group. Remaining 176 samples are labeled as pathological which indicate the existing of fetal distress. In this experiment, mentioned response variables between the features of LB, AC and FM graph will be different. The graphs are presented by Figs. 5-7.

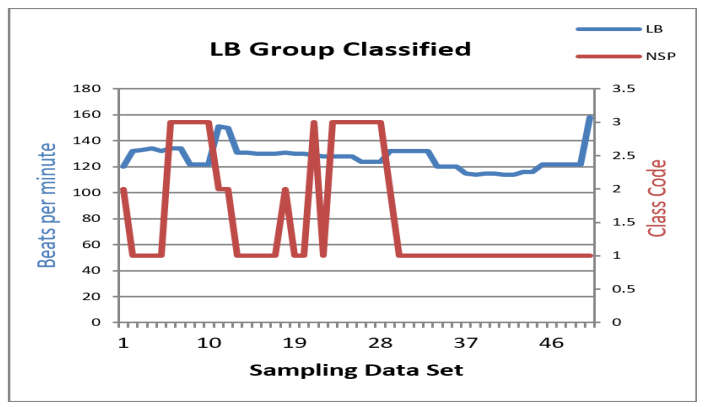

Fig. 5: LB groups Classified 


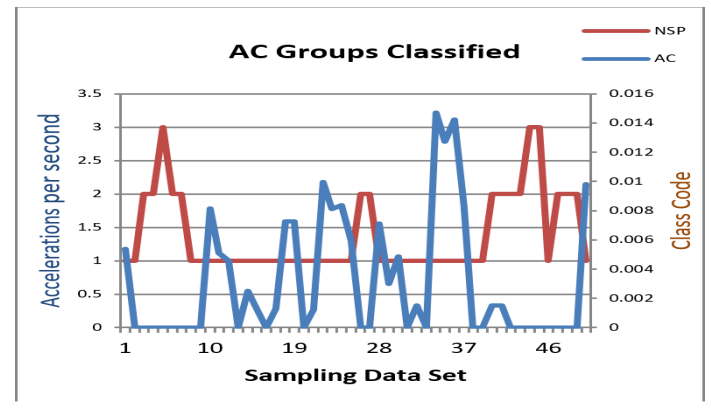

Fig. 6: AC Groups Classified

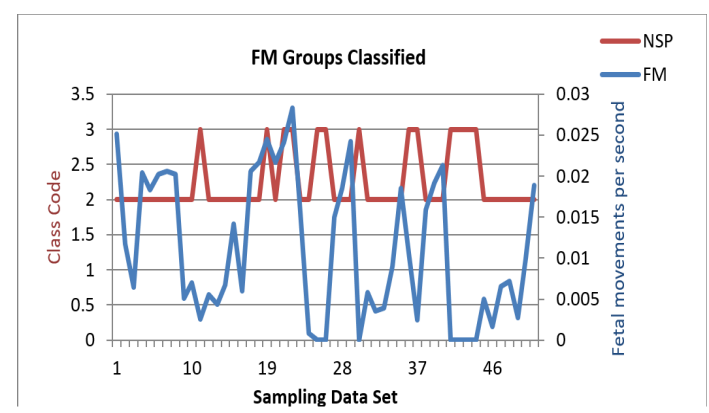

Fig. 7: FM Groups Classified

For this graph we choose randomly data .Every features we sampling 50 data. In this graph if we analysis this three features with response variable it's clearly represent the differences of them. LB features data is represented FHR baseline (beats per minute). Mentioned Fig. 4 shows the LB vs NSP (class code) variables figured. Similarly reaming two features AC and FM defined the FHR accelerations per second and fetal movements per second.

The cardiotocography enrolls the heart rate mutability, uterine compressions and fetal activities simultaneously during pregnancy. It gives a gynecologist knowledge about the cardiovascular system of fetal and the fetal activities [24]. Women who have bleeding, high blood pressure, premature labor or diabetes, CTG is specially recommended for them. An obstetrician can understand the CTG report through some key parameters.

+ Fetal heart rate Baseline (BL) - 110-150 beats every 60 seconds $(\mathrm{bpm})$ are in the range of physiological values. If the $\mathrm{BL}>150 \mathrm{bmp}$, that means the tachycardia and if the $\mathrm{BL}<110 \mathrm{bmp}$, that means the bradycardia.

+ Acceleration (ACC) - for at least 15 seconds rises $\mathrm{BL}$ upper than $15 \mathrm{bpm}$. In every 15 minutes the acceleration should happen at least double. Dur- ing the night time acceleration is regarded as pathological condition and at the same time it could be responded to fetal activity.

+ Deceleration (DCL) - for at least 15 seconds decrease BL higher than $15 \mathrm{bpm}$. Along with constrictions it may imply birth asphyxia.

\section{Conclusion}

Machine learning is one of the best choice for medical diagnosis and prediction. In this study, we are applied several machine learning techniques and $\mathrm{R}$ programming simulation software to accomplish this approach, classification of cardiotocography. After applying suitable machine learning technique when data set is trained, the prediction is acquired certain feature. This research methodology may help to related people who are working about embryology.

This study focused only on fetal conditions where the health status of both the mother and the fetus can be considered and studied in future. The nobility of this research is to trace the fetal status during pregnancy period especially for the complicated cases.

\section{References}

[1] Grivell, R. M., Alfirevic, Z., Gyte, G. M., \& Devane, D. (2015). Antenatal cardiotocography for fetal assessment. Cochrane Database of Systematic Reviews, (9).

[2] Cesarelli, M., Romano, M., \& Bifulco, P. (2009). Comparison of short term variability indexes in cardiotocographic foetal monitoring. Computers in biology and medicine, 39(2), 106118.

[3] Steer, P. J. (2008). Has electronic fetal heart rate monitoring made a difference?. In Seminars in Fetal and Neonatal Medicine (Vol. 13, No. 1, pp. 2-7). WB Saunders.

[4] Chamidah, N., \& Wasito, I. (2015). Fetal state classification from cardiotocography based on feature extraction using hybrid K-Means and support vector machine. In 2015 International Conference on Advanced Computer Science and Information Systems (ICACSIS) (pp. 37-41). IEEE.

[5] Magenes, G., Pedrinazzi, L., \& Signorini, M. G. (2004). Identification of fetal sufferance antepartum through a multiparametric analysis 
and a support vector machine. In The 26th Annual International Conference of the IEEE Engineering in Medicine and Biology Society (Vol. 1, pp. 462-465). IEEE.

[6] Cesarelli, M., Romano, M., \& Bifulco, P. (2009). Comparison of short term variability indexes in cardiotocographic foetal monitoring. Computers in biology and medicine, 39(2), 106118.

[7] Gribbin, C., \& Thornton, J. (2006). Critical evaluation of fetal assessment methods. et al. High risk pregnancy management options, 3rd edition. Philadelphia, USA: Elsevier.

[8] Spyridou, K. K., \& Hadjileontiadis, L. J. (2007). Analysis of fetal heart rate in healthy and pathological pregnancies using waveletbased features. In 2007 29th Annual International Conference of the IEEE Engineering in Medicine and Biology Society (pp. 1908-1911). IEEE.

[9] Parer, J. T., \& King, T. (2000). Fetal heart rate monitoring: is it salvageable?. American journal of obstetrics and gynecology, 182(4), 982987.

[10] Thacker, S. B., Stroup, D., Chang, M. H., \& Henderson, S. L. (2001). Continuous electronic heart rate monitoring for fetal assessment during labor. Cochrane database of systematic reviews, (2).

[11] Van Geijn, H. P., Jongsma, H. W., de Haan, J., \& Eskes, T. K. (1980). Analysis of heart rate and beat-to-beat variability: Interval difference index. American journal of obstetrics and gynecology, 138(3), 246-252.

[12] Safavian, S. R., \& Landgrebe, D. (1991). A survey of decision tree classifier methodology. IEEE transactions on systems, man, and cybernetics, 21(3), 660-674.

[13] Batra, A., Chandra, A., \& Matoria, V. (2017). Cardiotocography Analysis Using Conjunction of Machine Learning Algorithms. In 2017 International Conference on Machine Vision and Information Technology (CMVIT) (pp. 1-6). IEEE.

[14] Newman, D. J., Hettich, S. C. L. B., Blake, C. L., \& Merz, C. J. (1998). UCI repository of machine learning databases, 1998.

[15] Breiman, L., Friedman, J., Stone, C. J., \& Olshen, R. A. (1984). Classification and regression trees. CRC press.

[16] Timofeev, R. (2004). Classification and regression trees theory and applications. Master,
Humboldt University, Center of Applied Statistics and Economics.

[17] Ramla, M., Sangeetha, S., \& Nickolas, S. (2018). Fetal health state monitoring using decision tree classifier from cardiotocography measurements. In 2018 Second International Conference on Intelligent Computing and Control Systems (ICICCS) (pp. 1799-1803). IEEE.

[18] Sahin, H., \& Subasi, A. (2015). Classification of the cardiotocogram data for anticipation of fetal risks using machine learning techniques. Applied Soft Computing, 33, 231-238.

[19] Salzberg, S. L. (1994). C4. 5: Programs for machine learning by j. ross quinlan. morgan kaufmann publishers, inc., 1993.

[20] Quinlan, J. R. (1996). Improved use of continuous attributes in C4. 5. Journal of artificial intelligence research, 4, 77-90.

[21] Han, J., Kamber, M., \& Pei, J. (2012). Data mining: concepts and techniques, Waltham, MA. Morgan Kaufman Publishers, 10, 978-1.

[22] Witten, I. H., Frank, E. (2012). a Hall, Data Mining: Practical Machine Learning Tools and Techniques, 2nd ed. United States of America.

[23] Karabulut, E. M., \& Ibrikci, T. (2014). Analysis of cardiotocogram data for fetal distress determination by decision tree based adaptive boosting approach. Journal of Computer and Communications, 2(9), 32-37.

[24] Shah, S. A. A., Aziz, W., Arif, M., \& Nadeem, M. S. A. (2015). Decision trees based classification of cardiotocograms using bagging approach. In 2015 13th International Conference on Frontiers of Information Technology (FIT) (pp. 12-17). IEEE.

\section{About Authors}

Md. Zannatul ARIF received his B.Sc in Electrical and Electronics Engineering from International Islamic University Chittagong (IIUC), Bangladesh. He is presently continuing his master's degree in Electrical power system and its automation, North China Electric Power University (NCEPU), Beijing, China. Now, he is doing research under communication lab of North china electric power university. His research interests are machine learning, internet of things and Smart grid. 
Rahate AHMED received his B.Sc in Electrical and Electronic Engineering from International Islamic University Chittagong, Bangladesh. Currently, he is pursuing M.Sc in Nuclear and Energy Engineering at Jeju National University, Republic of Korea. He has over 8 publications and 4 patent His research area includes triboelectric nano generator, thermo magnetic generator, computer vision and AI based solar tracker, and active day lighting systems.

Umma Habiba SADIA received her Bachelor Degree in Business Administration from National University, Dhaka. Now, she is doing her master's Degree in School of Management and Economics from North China Electric Power University (NCEPU), Beijing, China. Her research interests include data Mining, Sustainable development and innovation management.
Mst Shanta Islam TULTUL received her MBBS degree from Faridpur Medical College under University of Dhaka, Bangladesh. Now she is pursuing her M.Sc in Medicine at Jeju National University, Republic of Korea. Her research interest includes microbiology, immunology, and bioinformatics.

Rocky CHAKMA received his B.Sc in electrical and electronics engineering (EEE) from International Islamic University Chittagong (IIUC), Bangladesh, also he has completed his master's degree from South China University of Technology, Guangzhou, China. At present time he is a faculty member of Electrical and Electronic Engineering (EEE) Department, University of Science and Technology Chittagong, Bangladesh. He has over 10 publications. His research interests include WSN, renewable energy and solar materials, Image processing.

"This is an Open Access article distributed under the terms of the Creative Commons Attribution License, 73 which permits unrestricted use, distribution, and reproduction in any medium provided the original work is properly cited (CC BY 4.0)." 